\title{
POLÍTICAS PÚBLICAS EN COLOMBIA DE GENERACIÓN DISTRIBUIDA EN NUEVAS FUENTES ENERGÉTICAS: \\ EL CASO DE LA GEOTERMIA Y LAS ZONAS NO INTERCONECTADAS.
}

\section{Public Policies in Colombia about Distributed Generation Systems in New Energy Resources.}

Geothermal case in non-connected areas

\begin{abstract}
Katherin Farietta Jiménez ${ }^{1}$
Universidad Santo Tomás-Bogotá
\end{abstract}

Fecha de recepción: 01.11.2013

Fecha de evaluación: 10.03.2014

Fecha de aprobación: 20.04.2014

\section{Resumen.}

La falencia en la prestación del servicio de energía en las áreas no interconectadas del país lleva a concluir que hace falta implementar políticas fuertes a nivel nacional y que se desarrollen por parte de los gobiernos locales para dignificar la calidad de vida de sus habitantes.

Además la firma y ratificación de tratados internacionales por parte de nuestro estado para diversificar la canasta energética, hace propicio el escenario de las zonas no interconectadas para su pleno y rentable abastecimiento mediante sistemas de generación distribuida con fuentes de energía limpias.

No obstante es necesario dejar de lados los intereses clientelistas de los particulares y que el gobierno central incentive la electrificación eficiente de las zonas que en nuestro mapa representan un porcentaje bastante considerable.

Palabras Clave: Geotermia, Energías alternativas renovables, generación distribuida, políticas públicas.

\section{Abstract}

\footnotetext{
${ }^{1}$ Abogada graduada con honores, Universidad Santo tomas debido a trabajo de grado, Marco Jurídico de la Geotermia para Uso Energético, sugerido por la Unidad de Planeación Minero energética - Upme con publicación en proceso para esta misma entidad. Investigadora independiente.
}

REVISTA VIA INVENIENDI ET IUDICANDI, VOL. 8, No. 2 
Shortcomings in the utility energy service in non-interconnected energy areas, evidences the necessity to implement stronger national policies to be developed for local governments in order to dignified living conditions from people who live in there.

Furthermore Colombia's international compromises to diversify energy sources, encourage to supply energy in a successfully and profitably way through clean energy sources.

Moreover it is necessary to get apart particular interests and rather central government promotes electrification in an efficient way on those non - interconnected perimeters that are part of a siginicative percentage on the Colombia`s map.

Keywords: Geothermal energy, Renewable Alternative Energies, Distributed Generation, Public Policies.

\section{Introducción: El Caso de la Geotermia y las zonas no interconectadas}

Por primera vez se estudiaron probabilidades de explotación de posos de geotermia para producción de energía eléctrica en el año 1989, por parte de la Organización Latinoamericana de Energía - OLADE en un contexto histórico cobre la toma de conciencia sobre las "inoportunidades" que desata el uso de energéticos convencionales, propulsado por los países potencia en respaldo y financiamiento a América Latina.

Dichos financiamientos llegaron a Colombia para encontrar resultados positivos en el tema de exploración de nuevas fuentes energéticas endógenas por parte de la mencionada Organización Latinoamericana de Energía y fueron retomados hacia el 2008, por una conocida empresa comercializadora con el apoyo de algunas entidades estatales. En los cuales se encontró propicia la explotación de una fuente completamente renovable y auto generadora, caracterizada por ser la única fuente alternativa renovable que funciona los 365 días del año, las 24 horas del día.

Al hablar de comercialización de nuevas fuentes energéticas, inevitablemente se habla sobre los problemas que desata su posicionamiento geográfico y el costo de su oferta, 
las cuales son escasas para nuestro actual nuestro ordenamiento jurídico, político y normativo, impidiendo que estas puedan competir en bolsa con rentabilidad debido a sus elevados costos de generación y transporte y para lo cual es conveniente hablar de generación in situ o generación local.

Por regla general la energía en la que se abastece energía en nuestro país es por medio del despacho desde un centro de administración y operación denominado “Centro Nacional de Despacho". Permitiendo la ley solamente en casos excepcionales que en algunos casos se pueda generar y comercializar energía de manera independiente.

A diferencia de Colombia, Estados Unidos permite y promueve el uso de generación descentralizada, por medio de regímenes de "Generación Distribuida" los cuales en este tiempo son una excelente alternativa para que se pueda promover energía a cortas distancias de manera confiable y rentable.

Debido a lo anterior el propósito de este artículo es contextualizar al lector sobre la reglamentación de las Energías Alternativas Renovables en el mundo, así como la forma en que se comercializa energía en nuestro país con el propósito de explorar nuevas fuentes de energía del tipo "No Convencional", desde un punto de vista beneficioso para los inversionistas y usuarios, así como en procura del cuidado del medio ambiente.

Para ello se exponen a groso modo los inconvenientes legales y reglamentarios que existen para incursión de modelos de generación distribuida y se explica porque estos facilitan la promoción de fuentes de energía limpias. Proponiendo a su vez la implementación de políticas públicas eficientes que susciten formas de uso, generación y distribución de energía en las zonas no interconectadas del país. 


\section{La Energía Geotérmica dentro de las Fuentes de Energía Renovables No Convencionales.}

"Si se clasifican las fuentes de energía, teniendo en cuenta su duración en el tiempo, estas pueden dividirse en fuentes convencionales y fuentes no convencionales. Siendo las segundas aquellas fuentes que tienen periodos de vida longevos y en algunos casos infinitos, que además de ser renovables contribuyen al desarrollo sostenible del medio ambiente y la economía, pues no afectan el medio ambiente, como sucede con las fuentes convencionales y sus emisiones de CO2 en la atmósfera"”

Entre las fuentes de energía limpias, se destacan la Energía Solar, la energía eólica, la energía hidroeléctrica, biomasa, mareomotriz y algunas otras. Pero la única fuente con disponibilidad ilimitada, todos los días del año y las veinticuatro horas del día con una potencia de abastecimiento extraordinaria se denomina "Energía Geotérmica".

La palabra "Geotermia" es de origen griego y proviene del sufijo "geo" que significa tierra y "termos" calor, es decir, el calor proveniente de la tierra. Esta es una energía de tipo termal, formada por un permanente flujo de calor desde el núcleo de la tierra, a través del manto y hacia la superficie terrestre. Es una energía completamente renovable y su calor es generado las veinticuatro horas del día durante todo el año, haciéndola casi infinita.

La energía geotérmica ha sido utilizada desde tiempos antiguos para calefacción y por cerca de 100 años para obtener energía eléctrica de manera continuada en el periodo moderno de la humanidad. Su potencial es inagotable en términos humanos y es comparable con la fuerza emanada de la energía solar.

\footnotetext{
${ }^{2}$ Formulación de un plan de Desarrollo para las Fuentes no Convencionales de Energía en Colombia, Consorcio Energético Corpoema, Volumen 1, página 1-1, Bogotá Diciembre de 2010.

REVISTA VIA INVENIENDI ET IUDICANDI, VOL. 8, No. 2

UNIVERSIDAD SANTO TOMÁS, BOGOTÁ

ISSN 1909-0528
} 
Fueron los romanos y los griegos quienes aprovecharon este fenómeno natural por primera vez, creando termas medicinales, baños públicos y usándolas para el calentamiento de sus casas. Fueron también ellos quienes propagaron el uso de esta energía a los continentes de Europa y América, expandiéndola a nivel mundial.

Se han encontrado registros de usos geotérmicos de más de dos mil años en China y existen ruinas romanas relacionadas con el aprovechamiento termal desde Siria hasta Inglaterra, doscientos años antes de Cristo ${ }^{3}$.

Pero solo hasta los siglos XVIII y XIX se descubrió el poder de condensación del vapor geotérmico gracias a los italianos, quienes innovaron en la producción de electricidad en Italia. Fue precisamente un italiano, el señor F.U. Hoefer ${ }^{4}$ director de la farmacia del Ducado de Toscana (Italia), quien encontró presencia de ácido bórico en los condensados de vapor geotérmico con una técnica que consistía en extraer el concentrado bórico con la quema de agua termal utilizando leña.

La geotermia empezó a ser una industria atractiva en el año 1827, con el señor Francois Larderel, el cual desarrolló un sistema para utilizar el calor de los fluidos en cada uno de los procesos de evaporación, directamente de la corteza terrestre. Así se remplazó la tradicional quema de madera, que provoca la deforestación de bosques, por la utilización de cada uno de los vapores que emanan directamente de la tierra.

De esta manera en el año 1829 entre 9 fábricas que producían energía geotérmica se destacaban: Castelnuevo, Sazzo, Serranazo, Lago y Monterrontondo.

\footnotetext{
${ }^{3}$ Financiamiento y regulación de las fuentes de energía nuevas y renovables, El caso de la Geotermia". Naciones Unidas, Comisión Económica Para América Latina y el Caribe. 1998

${ }^{4}$ http:// www.climatizaciongeotermica.com/geotermia/historia.html, consulta 20 de Febrero de 2012.

REVISTA VIA INVENIENDI ET IUDICANDI, VOL. 8, No. 2 
FARIETTA J., KATHERIN (2013): "POLÍTICAS PÚBLICAS EN COLOMBIA DE GENERACIÓN DISTRIBUIDA EN NUEVAS FUENTES ENERGÉTICAS: EL CASO DE LA GEOTERMIA Y LAS ZONAS NO INTERCONECTADAS”, VIA INVENIENDI ET IUDICANDI, VOL. 8, No. 2, PP.150-182

Cuatro años después en el barrio parisino de Grenelle ${ }^{5}$, se promovió por primera vez un sondeo en un pozo de 548 metros de profundidad, cuya construcción demoró ocho años. Además se constató la presencia de agua potable en el acuífero de arenas albienses de la Cuenca de París.

Japón también tomó la iniciativa en los caminos de la exploración de pozos geotérmicos en la ciudad de Beppu, localizada en la isla de Kyushu, instalando una planta experimental de 1 kilowatio para la generación parcial de energía eléctrica.

La técnica de aprovechamiento del vapor que emana de la tierra fue la que impulsó la creación de la primera empresa geotérmica en el mundo, llamada Darderello 1 (Italia), que luego de un tiempo aprovecharía el vapor geotérmico para producir energía, convirtiéndose en la primera comercializadora de energía geotérmica que monopolizaría el mercado europeo.

Las primeras formas importantes de producción de electricidad se deben, sin duda, a los italianos, quienes aprovecharon lo aprendido en Dardarello, poniendo a funcionar en el año de 1913 una central de 250 kilovatios, reutilizando además el vapor de baja presión para climatizar invernaderos, edificios industriales y residenciales.

Desde entonces, los países que han tomado el ejemplo para la instalación de redes usando energía geotermica han sido Francia, Italia, Hungría, Rumanía, Rusia, Turquía, Georgia, China, Estados Unidos e Islandia.

En la actualidad Alemania avanza en sus formas de explotación mientras que Suiza espera explotar una importante cantidad para el 2020. Por su parte Indonesia persigue la ambiciosa meta de convertirse antes del año 2025 en el mayor productor de

\footnotetext{
${ }^{5}$ http://www.uclm.es/cr/EUP-ALMADEN/aaaeupa/boletin_informativo/pdf/boletines/17/9.pdf. Consultado el 14 marzo de 2012.
}

REVISTA VIA INVENIENDI ET IUDICANDI, VOL. 8, No. 2 
electricidad geotérmica del mundo, con una capacidad total de 9.500 megavatios por hora.

El país que lidera la producción de energía geotérmica, es Estados Unidos, quien en la actualidad cuenta con 209 centrales en todo su territorio.

Además de los usos de generación para energía eléctrica, la energía geotérmica se utiliza hoy para calefacción (y refrigeración) de los edificios individuales, incluyendo oficinas, tiendas, pequeñas casas residenciales, así como fábricas, invernaderos y cultivos de piscicultura ${ }^{6}$.

\section{Propagación de Nuevas Fuentes de Energía en el Mundo.}

La idea de buscar ciertas fuentes energéticas que fueran más amigables con el medio ambiente no se presentó en un contexto de preocupación casual por daño al medio ambiente, sino que surgió en un contexto político crítico y devastador para la economía y la salubridad pública.

Con la segunda guerra mundial y las dos crisis energéticas de los años setenta, sobrevinieron secuelas ambientales, sociales y económicas que podían si bien no ser reparadas, al menos si podían mitigarse de manera considerable por medio de acuerdos y declaraciones con carácter vinculante de parte de cada uno de los estados implicados e interesados en la búsquedas de nuevos combustibles más accesibles.

El objetivo a trazar consistiría en reestructurar las políticas energéticas a nivel mundial buscando fuentes alternativas que generaran más fiabilidad ante un caso de desabastecimiento, así como de crisis energética y, que a la vez contribuyeran a mitigar la contaminación dejada en el medio ambiente.

\footnotetext{
${ }^{6}$ European Geothermal Energy Council, What is Geothermal? ,http://egec.info/about/ consultado el 12 de Abril de 2012

REVISTA VIA INVENIENDI ET IUDICANDI, VOL. 8, No. 2 
La primera de las crisis energéticas tuvo lugar el 17 de octubre de 1973, día en que la Organización de Países Árabes Exportadores de Petróleo tomó la decisión de adoptar medidas para no exportar petróleo a los países que apoyaron a Israel durante la guerra del Yom Kippur, entre los cuales se encontraba Estados Unidos y sus aliados de Europa Occidental y fue este acto el que desembocó una grave afectación en la economía europea y especialmente en la norteamericana, que estaba acostumbrada a poseer petróleo abundante y económico, además de fijar los precios del mismo en su moneda local.

En una crisis económica mundial el precio del dólar se devalúa, pero adicionalmente la presente tenía una particularidad, la devaluación del patrón oro que genero un efecto domino en las divisas latinoamericanas.

Estos aspectos conllevaron a una desvalorización del petróleo en todo el mundo, razón por la que Estados Unidos resultó favorecida respecto de sus competencias petroleras en Europa y Japón, pero que al mismo tiempo produjo una consecuente inseguridad política y financiera a nivel mundial, que fue transmitida a los países latinos que eran productores y comercializadores de petróleo en sus propias naciones y que tuvieron que afrontar pérdidas por la forzada crisis del patrón oro ${ }^{7}$.

La siguiente crisis de petróleo se presentó en el año 1979, pero esta vez Estados Unidos no se vio perjudicado, porque no dependía del combustible de los países orientales, sino que mantenía solidas relaciones comerciales con países latinoamericanos quienes se veían influenciados a vender a un menor costo ${ }^{8}$. Esta crisis se debió a la revolución Iraní y la guerra entre Irán - Irak que congeló la

\footnotetext{
${ }^{7}$ Medida necesaria que adoptó el gobierno norteamericano para provocar una caída del precio del oro de en los mercados internacionales, finalizando el sistema de Bretton Woods. Este era el sistema en el cual el oro era el patrón de valor del mercado internacional, adoptado por Estados Unidos como consecuencia de la segunda guerra mundial.

${ }^{8}$ Debido a la Deuda Externa de América Latina con Estados Unidos en los años ochenta.

REVISTA VIA INVENIENDI ET IUDICANDI, VOL. 8, No. 2

UNIVERSIDAD SANTO TOMÁS, BOGOTÁ

ISSN 1909-0528
} 
importación y determino que el precio del petróleo se disparara, provocando una segunda inflación a nivel mundial, que afecto especialmente las economías de Europa y Japón.

Fue así como surgió la necesidad de promover un uso más racional y eficiente en la canasta energética y de la diversificación de nuevas fuentes de energía ${ }^{9}$ por medio de la Agencia Internacional de Energía (AIE) en el año 1974. Esta agencia años más adelante se encargó de asuntos de crisis petrolera y estipulación de políticas entre estados adoptantes con la finalidad de asegurar energía confiable, accesible, pero sobretodo limpia.

Las secuelas ecológicas que causaron las dos guerras mundiales, fueron indudablemente otra de las causas por las que la organización internacional de las Naciones Unidas y algunos países potencias, tomaron la vocería en la búsqueda de alternativas más limpias y rentables a través de la firma de acuerdos internacionales vinculantes con el logro de objetivos concretos.

De allí surgieron importantes tratados legales para la mitigación de gases efecto invernadero, calentamiento global y promoción de nuevas fuentes de energía que consecuentemente llevo a cada uno de los Estados adoptantes a implementar medidas políticas y jurídicas acertadas para la diversificación de su canasta energética.

En los años siguientes a 1988 varios organismos y agencias de cooperación internacional, decidieron divulgar el uso de energías alternativas renovables no convencionales, como un asunto de interés mundial y por lo mismo empezaron las grandes cumbres contra el cambio climático en las cuales participaban y se hacían miembro países de todas las partes del mundo.

\footnotetext{
${ }^{9}$ Acosta M., AMYLKAR D, Regulación Internacional de las Energías renovables y de la Eficiencia Energética, Universidad Externado de Colombia, 2011.

REVISTA VIA INVENIENDI ET IUDICANDI, VOL. 8, No. 2

UNIVERSIDAD SANTO TOMÁS, BOGOTÁ

ISSN 1909-0528
} 


\section{Promoción de nuevas fuentes en Colombia - La Geotermia}

En la promoción y fomento de energías alternativas el Plan Nacional de Desarrollo del periodo 2010 - 2014 en su artículo 105 preceptúa, un posterior desarrollo político por parte del gobierno para impulsar las fuentes de energías alternativas, sin sentar unas bases fijas para una participación eficaz de las mismas. Dejando por fuera las formulaciones y recomendaciones para el tratamiento de dichas políticas públicas en la consultoría que realizo el consorcio energético Corpoema en conjunto con la Unidad de Planeación Minero Energética - Upme en tres volúmenes de investigación detallada del año $2010^{10}$.

Lo que se ha desarrollado más bien de manera muy ambigua para el tema de la promoción de energías alternativas renovables, proviene del primer encuentro vinculante para nuestro país en la convención marco de naciones unidas para el cambio climático y que se despliega legalmente en la ley 697 y su decreto reglamentario. Pero que aun así se sigue quedando corto debido a planes estratégicos detallados.

Debido a que la Convención Marco de las naciones unidas contra el cambio climático no tenía fuerza vinculante para los Estados adoptantes, ni tampoco contenía objetivos contundentes a plazo específicos, se acordó un documento adherente que contuviera decisiones más precisas y obligatorias.

Colombia no solamente firmó e incorporo a nuestro ordenamiento jurídico la Convención Marco de las Naciones Unidas para el Cambio Climático mediante Ley 164 de 1994 sino su adherente protocolo de Kioto.

\footnotetext{
${ }^{10}$ Consorcio Energético Corpoema, Formulación de un Plan de Desarrollo para las Fuentes no Convencionales de Energía en Colombia,

http://www.upme.gov.co/Sigic/DocumentosF/Vol_1_Plan_Desarrollo.pdf, consultado el 5 de febrero de 2012.
}

REVISTA VIA INVENIENDI ET IUDICANDI, VOL. 8, No. 2 
Dicho protocolo se agregó mediante ley 697 de 2001 y es un acuerdo internacional de suma importancia en el desarrollo de políticas y normas con respecto a fuentes energéticas limpias que centra su importancia en la rentabilidad de las fuentes, a la vez que al ser inherente al concepto de desarrollo sostenible y a la vez reconoce la importancia a la distribución de la ecología.

La ley 697 de 2001, más conocida como "La Ley que fomenta el uso racional y eficiente de la energía - URE" busca otorgar un tratamiento especial al consumo energético, regulándolo como un asunto de interés social, público y de conveniencia nacional en procura de asegurar abastecimiento energético pleno y accesible pero a la vez promoviendo el uso de Energías no convencionales de manera sostenible con el medio ambiente y los recursos naturales.

A esta ley la reglamenta el Decreto 3683 de 2003, el cual básicamente crea una "Comisión Intersectorial para el uso racional de la energía y la promoción de nuevas fuentes" además de promover e incentivar la investigación de las mismas.

\section{Políticas Energéticas en el Estado de California.}

El estado de California cuenta con un mercado energético eficiente, gracias a la restructuración de sus políticas públicas y la implementación de marcos jurídicos coherentes, claros y con incentivos hacia fuentes energéticas limpias, pero ello no siempre fue así.

\section{A. Comercialización de energía en California}

Hacia el año 2000 una fuerte crisis en el sector energético generó el cierre de la bolsa y la quiebra de los agentes comercializadores y generadores, que se fueron a perdidas como resultado de las de políticas no relacionadas con la normatividad existente para 
la época. A ello se sumó una fuerte subida en la demanda de energía producto de la dependencia en la importación de energía, además de que el fenómeno del niño ocurrido para el año dos, generó un desabastecimiento en los embalses del estado californiano que dependía principalmente de otras regiones.

Fue así como años posteriores a esta crisis, se pretendió crear un proceso de "Desregularización parcial del mercado energético"11 que pretendía implementar un proceso de liberalización en la etapa de comercialización que buscaba una variedad en la oferta al consumidor, en el momento en el cual este eligiera su agente proveedor. De la misma manera este proceso se pretendía también una libre competencia en el ejercicio de comercialización, que hasta el momento estaba en manos de las tradicionales empresas distribuidoras ${ }^{12}$.

Por un largo periodo de tiempo, el mercado de la distribución de energía en el estado de California se administra a través de un sistema centralizado que mediante un conjunto de grandes centrales de generación conectadas a redes con alta capacidad de transporte, que interconecta vastas regiones y permite el transporte de energía hacia cualquier centro de consumo que se despache mediante la administración de una bolsa de energía diaria.

La actividad de suministro central de la energía en el estado de California, consiste en la compra y venta mediante un agente denominado "California Power Exchange" para que posteriormente esta sea despachada a cargo de otro agente denominado "Independent System Operator" por medio de unas redes eléctricas instaladas para tal fin.

\footnotetext{
${ }^{11}$ Pontificia Universidad Católica de Chile, Escuela de Ingeniería, Estudio Comparativo de las Crisis Eléctricas en Chile, California y Brasil; Aspectos Relevantes para el Nuevo Marco regulatorio Chileno, http://web.ing.puc.cl/ power/paperspdf/ariztia.pdf, revisado el 26 de mayo de 2012.

12 Las tres grandes empresas distribuidoras eran para el año 2000, Pacific Gas and Electric Company, Southern California Edison Company y San Diego Gas and Electric Company,
} 
Quienes participan en la segunda fase del suministro energético son los las empresas generadoras de energía, los agentes comercializadores y el usuario final, pero como se verá más adelante en el mercado de energía en Estados Unidos los mismos generadores de la electricidad pueden ser a la vez agentes comercializadores que proveen de manera local a sus usuarios finales, mediante una forma descentralizada se denominada "Generación Distribuida".

B. Generación distribuida en California. (Coexistencia de generación centralizada y la generación distribuida.

La generación distribuida consiste en la generación o el almacenamiento de energía eléctrica a pequeña escala de la forma más cercana al centro de carga pero que otorga la posibilidad de interactuar (comprando o vendiendo) con la red eléctrica y considerando la máxima eficiencia energética ${ }^{13}$.

Esta forma de despacho local era antiguamente la elegida para provisionar suministro a nivel nacional, pero con el tiempo esto fue remplazado por sistemas de generación central debido a necesidades de control sobre el abastecimiento. Sin embargo debido a la fuerte crisis petrolera que afectó la economía norteamericana en los años ochenta, se creó la necesidad de retomar el método antiguamente utilizado, ya que este facilitaba la confiabilidad del suministro local en los diferentes condados de los estados pertenecientes al país norteamericano.

La época de los años ochenta para los Estados Unidos, llevo inmersa una profunda crisis política y económica, debido a las complicaciones en el acceso al petróleo, ya que para esta fecha dicho combustible empezaba a ser escaso. Para esta época los

\footnotetext{
${ }^{13}$ Comisión de Regulación de Energía y Gas, La Generación distribuida y su posible integración al sistema interconectado nacional, http://apolo.creg.gov.co/Publicac.nsf/0/7128194c8ff15d7d0525785a007a71f5/\$FILE/CIRCULAR0532009\%20ANEXO.pdf, Consultado el de marzo de 2012
}

REVISTA VIA INVENIENDI ET IUDICANDI, VOL. 8, No. 2 
países que poseían importantes reservas eran los países latinoamericanos y los países de oriente medio, pero con respecto a estos últimos, Estados Unidos tenía una relación política bastante deteriorada ${ }^{14}$

Este aspecto llevo a buscar alternativas existentes que hasta el momento no eran utilizadas para generar energía eléctrica y métodos que lograran un uso más eficiente en los tiempos de ofertas en pico, en puntos de generación, distribución y transporte locales.

Mientras que en los sistemas de despacho central de energía, un sistema de grandes líneas centrales se interconectan a otras redes con alta capacidad de transporte que llegan hasta vastas regiones, accediendo el paso energía a "casi" cualquier centro de consumo, la generación distribuida consiste en un sistema energético descentralizado mediante el cual se permite a pequeños y grandes generadores locales proporcionar energía a clientes a cortas distancias, instalando y conectando las unidades de generación de energía de manera directa a la red de distribución o de la manera próxima posible al cliente.

Pero para hacer el mercado de la generación local más eficiente, en especial a determinadas horas pico, se ha fusionado el aprendizaje tecnológico con el de la producción de energía local mediante un sistema energético conectado a través de redes inteligentes monitoreadas, el suministro energético que permite controlar niveles precisos de descarga que aprovechen todo lo posible el recorrido total de la energía considerado tanto mecanismos tecnológicos, como precios consistentes en tarifas de electricidad basadas en el tiempo de medición neta, contadores inteligentes y estándares de interconexión uniforme. Sobre los cuales se hablará más adelante en este artículo.

\footnotetext{
${ }^{14}$ Crisis energéticas años 1973 y 1979

REVISTA VIA INVENIENDI ET IUDICANDI, VOL. 8, No. 2

UNIVERSIDAD SANTO TOMÁS, BOGOTÁ

ISSN 1909-0528
} 
La gran ventaja de este sistema es además que puede ser de propiedad y operación de terceros, empresas de servicios públicos o clientes, con capacidad de generación y distribución y lo más importante es que beneficia a todos los usuarios que se encuentran en zonas muy apartadas en donde el sistema a veces no es capaz de abastecer energía a ciertos usuarios de manera constante o quienes se ven afectados en el suministro en horas pico, así como aquellos que se valen de sistemas de cogeneración.

Para el caso de Estados Unidos estos sistemas son muy usuales en áreas municipales y campus universitarios.

\section{Régimen de energía eléctrica en Colombia}

\section{A. Régimen central}

El régimen de despacho central de energía en Colombia, consiste en la administración y operación de las actividades de generación, transmisión, distribución y comercialización, desde un punto central.

La operación del Sistema Interconectado Nacional se encuentra a cargo de la empresa filial de ISA $(X M)^{15}$ con la participación de las diferentes empresas de servicios públicos por medio del mercado spot mediante subastas diarias y con base en tarifas fijadas por la Comisión de Regulación de Energía y Gas - CREG.

Los usuarios del mercado central de energía se clasifican en usuarios regulados (mercado minorista) o no regulados (mercado mayorista) haciendo referencia para los primeros, a pequeños consumidores ${ }^{16}$ y el para el segundo, al mercado mayorista, los cuales son aquellos que consumen energía en un monto superior a $0.1 \mathrm{MW}$ o con una

\footnotetext{
${ }^{15} \mathrm{XM}$ además administra las transacciones internacionales de electricidad con Ecuador

${ }^{16}$ Representan el $75 \%$ del mercado.
}

REVISTA VIA INVENIENDI ET IUDICANDI, VOL. 8, No. 2 
demanda superior a $55 \mathrm{MWh}$-mes ${ }^{17}$, a través de contratos de suministro a largo plazo entre el ente generador y el cliente con la administración de XM.

\section{B. Zonas no Interconectadas}

Las Zonas No Interconectadas son aquellas áreas geográficas como municipios, corregimientos, localidades y caseríos que no están conectadas al Sistema Interconectado Nacional ${ }^{18}$, ni tampoco hacen parte del mercado mayorista y por ello no participan en el mercado diario Spot.

Estas áreas abastecen de energía a sus usuarios mediante pequeñas plantas locales que casi siempre utilizan diésel abastecimiento solo a ciertas horas del día y ocupan una extensión de aproximadamente el $66 \%$ del territorio nacional $(754.000 \mathrm{Km} 2)$ en los cuales habitan alrededor de 1.500 .000 habitantes por Km2 y se subdividen en áreas Continentales y áreas Insulares que pertenecen a las regiones del Caribe, Pacifico, Orinoquia y Amazonia.

Las áreas continentales comprenden los departamentos de Amazonas, Antioquia, Arauca, Caquetá, Casanare, Cauca, Chocó, Guainía, Guaviare, Meta, Nariño, Putumayo, Vaupés y Vichada. Mientras que las insulares hace referencia a las islas de San Andrés y Providencia.

\footnotetext{
${ }^{17}$ Representan el 25 del mercado.

${ }^{18}$ Colombia, Comisión de Regulación de Energía y Gas, 2008, ( 2008, 22 de Diciembre), "resolución 160 de 2008, Por la cual se definen las reglas para verificar la existencia de los motivos que permiten la inclusión de áreas de servicio exclusivo en los contratos y los lineamientos generales y las condiciones a las cuales deben someterse ellos, para la prestación del servicio público domiciliario de energía eléctrica en el Archipiélago de San Andrés, Providencia y Santa Catalina", en el diario Oficial, núm. 47.214, 26 de diciembre de 2008, Bogotá.
} 
Las zonas no interconectadas tienen un régimen diferente para el caso del sistema interconectado nacional, por esa razón XM no rige su operación ni la administración de los contratos que se celebren y en cambio se le ha encomendado una labor de promoción de soluciones energéticas al Instituto de Planificación y Promoción de Zonas Energéticas para las Zonas No Interconectadas - IPSE, quien fomenta alternativas para una electrificación eficaz.

Para el abastecimiento de energía en estas zonas se hace uso generalmente Diésel, Pequeñas Centrales Hidroeléctricas y Sistemas de Paneles Solares, que para el caso del primero contaban con 1075 plantas que generaban electricidad con una capacidad de 199, 629,3 kW, para el año 2005. Mientras que para el caso de las centrales hidroeléctricas existían solamente tres plantas (Cauca, Choco, Costa Atlántica) con una Capacidad Instalada de $2584 \mathrm{~kW}$ y los paneles fotovoltaicos, 52 en total para estas áreas, generaban energía con una capacidad de $148.48 \mathrm{Kw}$.

El Ipse y la Upme en su papel de planeación hacia un uso eficiente de la energía, han intentado promover la explotación de nuevas fuentes de energía para estas zonas apartadas del país, mediante investigaciones y estrategias contundentes que avancen en este campo, sin que aún haya lineamientos legales fuertes para materializar estos esfuerzos.

Como se vio renglones arriba se ha avanzado en la investigación de la energía geotérmica, encontrándose reservorios potentes para abastecimiento. Pero para ello es necesaria una reglamentación clara que permita la distribución localizada mediante incentivos eficaces para el uso de fuentes limpias y unas políticas públicas coordinadas con el gobierno central y los gobiernos locales en promoción de electrificación eficaz en las zonas apartadas del país.

Respecto de su régimen tarifario, este es fijado con base a unos parámetros fijados por la Comisión de Regulación de Energía y Gas - CREG. 


\section{Nuevas políticas públicas}

A. Falencias en las Policías Actuales para las Zonas no Interconectadas.

Las ventajas de la electrización en zonas rurales como primer paso para el desarrollo de políticas públicas eficientes permiten la modernización y el avance en otros aspectos sociales en estas zonas de evidente atraso social, pero además contribuyen con el tema del uso de fuentes de energía limpias al ser escenarios que permiten la protección ambiental y la reducción de costos tarifarios usualmente gravados como producto de combustibles tales como el diésel.

Con la finalidad de poder combinar los beneficios de energías limpias a costos rentables en las áreas periféricas del país, es necesario establecer la fijación de políticas públicas que propongan un desarrollo metodológico que responda a las situaciones geográficas y demográficas que presentan estas áreas producto de los esquemas centralistas, si se tiene en cuenta que tradicionalmente en Colombia ha pensado que se requiere contar con la disponibilidad de un sistema de información centralizado, ya que este identifica de manera confiable a los usuarios del servicio de energía eléctrica con referencia al tipo de usuario, ubicación y el correspondiente agente prestador del servicio con base a juicios de confiabilidad en el abastecimiento, despacho y demanda precisa. Sin embargo, este modelo permite evidenciar a una política centralista y tradicional que no tiene en cuenta a todos los actores que habitan las diferentes regiones del país y que por consiguiente conlleva a replantear las actuales políticas públicas, para ser cambiadas por proyectos que permitan modelos de generación descentralizada al menos para las áreas no interconectadas del país.

Para efectos prácticos de este artículo "Política Publica" es el estudio de las situaciones (necesidades y logros) de un determinado grupo, así como la participación del mismo 
FARIETTA J., KATHERIN (2013): "POLÍTICAS PÚBLICAS EN COLOMBIA DE GENERACIÓN DISTRIBUIDA EN NUEVAS FUENTES ENERGÉTICAS: EL CASO DE LA GEOTERMIA Y LAS ZONAS NO INTERCONECTADAS”, VIA INVENIENDI ET IUDICANDI, VOL. 8, No. 2, PP.150-182

en un determinado plan que implemente metas a corto, mediano y largo plazo ${ }^{19}$ mediante Planes Territoriales de Desarrollo ${ }^{20}$ y con la administración de los respetivos gobiernos locales.

Por medio de ellas para que en el caso de la electrificación de zonas aledañas, y por medio de proyectos locales de industrialización se pueden lograr excelentes obras de electrificación que atiendan las necesidades de modernización y desarrollo en aquellas áreas que aún no cuentan con un servicio público digno de nuestro actual estado social de derecho y para lo cual se necesita promoción, inversión y reglamentación en procura también de la conservación y el respeto de tradicionales criterios históricos en relación a las reservas de riqueza biótica y ambiental con las que estas áreas cuentan.

Valga la pena redundar en el concepto de la palabra "modernización", el cual no lleva implícito el monte de fábricas de producción que destruyan las actuales condiciones que hacen de la gran mayoría de estas zonas lugares privilegiados y de conservación, sino que hace referencia a la garantía para sus habitantes sobre el acceso a la educación, a la salud y a las oportunidades de empleo correlacionados con sistemas de electrización confiable, continua y a un precio razonable con base a los magníficos posicionamientos geográficos en las que estas se encuentran y en fomento de oportunidades de educación, trabajo e ingresos locales como el ecoturismo.

En estas áreas es idóneo el uso de energías alternativas renovables que hasta el día de hoy en nuestro país no han sido integradas a procesos exitosos de comercialización ni a incentivos de tipo tributario. Tampoco han estado gestionadas a nivel de políticas de desarrollo energético local que les permita hacer del acceso a la energía un asunto

${ }^{19}$ Simposio Instituto de Desarrollo de Recreación y Deporte - IDRD, Que y para que las políticas públicas ${ }_{2}$

http://www.bogotamasactiva.gov.co/files/u1/Qu\%C3\%A9\%20y\%20Para\%20qu\%C3\%A9\%20sirven\%20las\%20Pol\% C3\%ADticas\%20Andr\%C3\%A9\%20Noel\%20Roth.pdf, Consultado el 15 de Marzo de 2013.

20 Colombia, Congreso de Colombia, año 1994, (1994, 15 de Julio) Ley 152 de 1994, Por la cual se establece la ley orgánica del plan nacional de desarrollo, en el diario oficial núm., 41.454, 15 de Julio de 1994.

REVISTA VIA INVENIENDI ET IUDICANDI, VOL. 8, No. 2 
confiable y razonable sin tener que competir en una bolsa central, debido al uso de combustibles costosos para estas regiones ${ }^{21}$ y a falencias respecto a la medición del consumo energético. Así como a los vacíos de regulación en la comercialización de pequeñas plantas que conlleva a que el costo que tiene que pagar el usuario final sea elevado, por la falta de contadores de energía o por el no mantenimiento en los ya instalados y que complica la medición del gasto para calcular el precio real por consumo, establecimiento un valor fijo al consumidor, según tipo de usuario o punto de conexión a la red.

Sin embargo existen otros problemas particulares de los entes prestadores que agravan la situación en estas regiones del país.

Según lo declarado por la Superintendencia de Servicios Públicos ${ }^{22}$ además de estar frente a un servicio oneroso, debido a la ausencia de mecanismos eficientes, el tema de la corrupción y la ilegalidad se hace evidente gracias a la violación de las normas contempladas en la ley 142 de 1994 en lo referente a Contratos de Condiciones Uniformes, así como al régimen jurídico para la creación de empresas de servicios públicos, los modos de estratificación estipulados en la misma ley 142, son otro problema, ya que que no están siendo tenidos en cuenta para la debida clasificación dentro de estas regiones conforme a los estipulado en la ley y lo establecido por el Ipse.

Estas situaciones conllevan a que la continuidad en la prestación del servicio eléctrico en un porcentaje general de cobertura por regiones, corresponda para a un $73 \%$ para la región de la Amazonía, para la región Pacífica a un 74\% y para la región del Atlántico al $45 \%$ con referente a la población ${ }^{23}$ y en términos horarios corresponda a un

\footnotetext{
${ }^{21}$ Diesel

22 Colombia, Comisión de Regulación de Energía y gas, 2003 ( 200325 de Septiembre de 2003), Resolución 073, Bases Conceptuales para la Regulación de las Prestación del Servicio de Electricidad en las Zonas No Interconectadas, Septiembre 25 de 2003

23 Ibídem.
}

REVISTA VIA INVENIENDI ET IUDICANDI, VOL. 8, No. 2

UNIVERSIDAD SANTO TOMÁS, BOGOTÁ

ISSN 1909-0528 
aproximado de 4 a 7 horas por día en las áreas de Mitú y Puerto Inírida, permitiendo concluir que pese al reajuste político y económico que permitió la expansión de la cobertura energética debido la crisis energética que vivió nuestro país a comienzos de los años noventa, aún faltan estrategias claras para abastecer de energía a las zonas que no están conectadas a la red central y que representan un porcentaje geográfico bastante relevante en el mapa de la nación, si se tiene en cuenta que el territorio tiene una connotación directa con la economía de un país y por ende es necesario ejercer actos tendientes a encontrar oportunidades para el desarrollo y aprovechamiento en cada una de las áreas geográficas de nuestro país en busca de su desarrollo.

Como ya es sabido el modelo tomado por el presidente cesar Gaviria en el año 1993 es producto de la globalización e internacionalización económica intrínsecamente relacionada con la garantía de que el estado asegura la prestación de todos los servicios públicos a los habitantes del territorio en condiciones óptimas y rentables, excepcionalmente por medio de sus entidades territoriales ${ }^{24}$ y más bien por entes de derecho privado o mixto, acatando, las principales funciones otorgadas a nivel legal por las leyes 142 y 143 del año 1994 referentes a las de regulación, subsidio a usuarios de menores recursos, planificación, vigilancia y establecimiento de políticas generales del sector $^{25}$.

Medidas todas estas planeadas en procura de necesidades no geográficas sino demográficas y que a simple vista cobra sentido.

B. Hacia unas políticas de desarrollo y adelanto

\section{Las zonas aledañas, La Energía Geotérmica y la Generación Local}

\footnotetext{
${ }^{24}$ Solo excepcionalmente

${ }^{25}$ Plan Indicativo de Expansión de Cobertura del Servicio de Energía Eléctrica, Unidad de Planeación Minero Energética - UPME, http://www.upme.gov.co/Docs/Plan_Expansion_Cobertura_Energia.pdf, consultado el 15 de Agosto de 2013.
}

REVISTA VIA INVENIENDI ET IUDICANDI, VOL. 8, No. 2 
El desarrollo de políticas públicas eficientes para la electrificación eficiente y racional en zonas rurales interconectadas a través del uso de fuentes de energía limpias permite la modernización y el avance social para los habitantes de estas regiones del país.

A través de soluciones energéticas integrales basadas en biocombustibles renovables, que están disponibles en las zonas no interconectadas a la red, con la capacidad de surtir las demandas requeridas, se puede cubrir la demanda no abastecida por la generación centralizada mediante sistemas de rentabilidad y facilidad en el aprovechamiento de fuentes energéticas limpias.

Para ello es necesario que el gobierno central promueva políticas públicas y por medio de los gobiernos locales se ejecuten proyectos en procura de un servicio eléctrico continuo y confiable que atienda a criterios de modernización y desarrollo, que a la vez promocionen el uso de energéticos renovables y económicamente más rentables que los actualmente existentes mediante proyectos de inversión y expansión para la electrificación, que conlleve además a una debida reglamentación que garantice el respeto a la historia, tradición y costumbres que caracterizan a estas zonas de importantes reservas de riqueza biótica y ambiental.

Valga la pena redundar en el concepto de la palabra "modernización" que para nada lleva implícito el concepto de construcción de fábricas de producción o grandes urbes que destruyan las actuales condiciones, que hacen de la gran mayoría de las Zonas No Interconectadas lugares privilegiados y de conservación. Se atiende entonces a encontrar esquemas que garanticen el acceso a la educación, la salud y a las oportunidades de empleo que necesitan los habitantes de estas regiones.

Los magníficos posicionamientos geográficos podrían ser tomados para crear importantes centros de educación superior públicos y privados que estudiaran las especies y ecosistemas que presentan regiones selváticas como la amazonia, el 
Guaviare y el choco las que a su vez, otorgarían nuevas oportunidades de empleo. Así como la creación de importantes centros turísticos ecológicos que estuvieran en sintonía con el concepto de conservación y preservación podrían ser creados en algunas de estas áreas, abriendo paso al turismo y al conocimiento de quienes no conocemos lo que característico de estas regiones, además

Es de suma importancia tener en cuenta el papel tan privilegiado que juegan las energías alternativas renovables en estas áreas, pero que al no estar incentivadas se encuentran en un proceso de estancamiento debido a la ausencia de una debida planeación de proyectos locales para su ejecución, apoyo y reconocimiento legal pero sobre todo por falta de apoyo del gobierno central.

Estos puntos podrían ser aprovechados para la generación de sistemas descentralizados de energía conectados a plantas de generación de energías renovables como la geotérmica, denominados "sistemas de generación distribuida" en la cual pudiera intervenir prácticamente cualquier agente que tuviera una planta de energía renovable y cualquier usuario que necesitara del abastecimiento energético.

Pero no como se está haciendo en la actualidad, sino con un reconocimiento específico propio expedido mediante una reforma de lo que hoy se conoce como al ley 142 y la 143 de 1994 referente al régimen de servicios públicos y régimen de energía en donde los actores energéticos al igual que ahora, competirían con base a parámetros de oferta y demanda regulados por la Creg por medio de incentivos tributarios estipulados en la ley y mediante una forma de despacho descentralizado debidamente regulado, en procura de un mercado atractivo y beneficioso para los diferentes agentes inversionistas de la cadena energética, así como para el usuario final.

Como se analizó líneas atrás la prestación del servicio eléctrico en estas áreas es ineficiente y costoso, y por lo tanto ha desencadenado en la migración de muchos de sus habitantes a áreas de asentamiento urbano, en el muchas veces la situación para 
ellos no mejora y que lleva a concluir que dentro de las agendas políticas de los diferentes gobiernos a nivel nacional y local, el tema del desarrollo en áreas periféricas no ha sido un eje central, quizás por razones de porcentajes a nivel poblacional y no geográfico, si se tiene en cuenta que el porcentaje total del territorio para estas zonas supera el $65 \%$, permitiendo evidenciar un desarrollo de modernización en estas áreas.

Vale la pena recordar que el artículo 365 de nuestra constitución preceptúa que es deber del Estado el garantizar la prestación eficiente de "todos los habitantes del territorio nacional" de conformidad con lo desarrollado en la ley, en lo referente a los principios de equidad ${ }^{26}$ y con un nivel de cobertura ${ }^{27}$ mínimo en el tema de los servicios públicos domiciliarios, por ello en el régimen de regulación actual se deben incluir esquemas diferenciales de prestación del servicio en generación, distribución y comercialización, con base a criterios de calidad, continuidad y atención del servicio que con antelación deben estar en primera línea dentro de las agendas de los gobiernos nacionales.

\section{Resultados de Reservorios Geotérmicos en Colombia}

Siendo la energía geotérmica una fuente de energía limpia que se explotara próximamente en Colombia capaz de bastecer a las zonas aledañas del país que no cuentan con sistemas de electrificación confiable, vale la pena destacar lo referente a los resultados para su uso en Colombia.

En 1981 la Organización Latinoamericana de Energía - OLADE realizó un estudio de reconocimiento de los recursos geotérmicos en Colombia, en el marco de un convenio

\footnotetext{
${ }^{26}$ Ley 142 de 1994, articulo 6

${ }^{27}$ Ley 142 de 1994, articulo 3.

REVISTA VIA INVENIENDI ET IUDICANDI, VOL. 8, No. 2

UNIVERSIDAD SANTO TOMÁS, BOGOTÁ

ISSN 1909-0528
} 
con el Instituto Colombiano de Energía Eléctrica - ICEL, financiado por la Comunidad Económica Europea ${ }^{28}$

El propósito de la investigación fue el de seleccionar áreas de interés geotérmico prioritario existentes en las tres cordilleras colombianas como base técnica para una posterior planificación y programación racional de desarrollo de fuentes geotérmicas.

En la Cordillera Occidental, en el área sur - norte se encuentran Los volcanes Chiles, Cerro Negro y Tufiño, así como el volcán Azufral.

Los Volcanes Chiles, Cerro Negro y Tufiño arrojaron los resultados que más se destacaron, por presentar rasgos de calor relativamente cerca a la superficie, así como fuentes termales de alto interés y posible presencia de rocas en profundidad que indican la presencia de un reservorio geotérmico ${ }^{29}$. Por su parte el volcán Azufral impactó positivamente por su "un vulcanismo de gran evolución, fuentes termales superficiales y fragmentos rocosos hidrotermalizados de tipo Vulcano-sedimentario que indican la existencia de un reservorio geotérmico de alta temperatura probablemente cubierto por rocas volcánicas terciarias que actúan como roca impermeable”.

Lo encontrado en la Cordillera Central, al estudiarse de sur a norte las áreas de los volcanes Doña Juana, Sotará, Puracé y Huila, indicaron reservorios geotérmicos sin certezas absolutas.

En la Cordillera Oriental se identificó como área importante la de Paipa, que "es la única situada fuera de la región volcánica principal, en un ambiente geológico

\footnotetext{
${ }^{28}$ Proyecto plan nacional de desarrollo, fuentes de energías no convencionales, volumen 2 , http:// www.upme.gov.co/Sigic/DocumentosF/Vol_2_Diagnostico_FNCE.pdf, Diciembre de 2012.

29 Ibídem.

REVISTA VIA INVENIENDI ET IUDICANDI, VOL. 8, No. 2 
FARIETTA J., KATHERIN (2013): "POLÍTICAS PÚBLICAS EN COLOMBIA DE GENERACIÓN DISTRIBUIDA EN NUEVAS FUENTES ENERGÉTICAS: EL CASO DE LA GEOTERMIA Y LAS ZONAS NO INTERCONECTADAS”, VIA INVENIENDI ET IUDICANDI, VOL. 8, No. 2, PP.150-182

sedimentario y la existencia de fuentes termales de alta temperatura en superficie sugeriría su calentamiento por algún cuerpo magmático en estado de latencia ${ }^{30}$.

En 2008 la compañía estadounidense Boston Pacific retomó las investigaciones ya adelantadas por OLADE y preseleccionó algunos sitios que pueden ser aprovechados de manera satisfactoria en Colombia. Entre estos se encuentra, la zona de influencia del Nevado del Ruíz y los volcanes Tufiño, Chile y Cerro Negro, en la frontera con el Ecuador.

Fue entonces cuando Isagen ${ }^{31}$ mostró su interés por el desarrollo e investigación de estos reservorios geotérmicos, emprendiendo un reto para la industria energética en el país denominado "Proyecto Geotérmico Binacional Tufiño chiles cerro negro" en honor al nombre de los reservorios geotérmicos ${ }^{32}$ Este proyecto en la actualidad se encuentra agotando la "Etapa de Factibilidad 33 " para asegurar la viabilidad y la posible área de cobertura de abastecimiento energético al norte de Ecuador y las zonas aledañas al departamento de Nariño y Choco.

3. Límites actuales a la generación descentralizada.

Hoy en nuestro país contamos con un sistema centralizado que se alimenta principalmente de fuentes hídricas y energías convencionales que pretenden abastecer la totalidad del territorio nacional por un sistema interconectado, sin lograr abastecer un considerable porcentaje del territorio en Colombia, descartando modelos de generación, distribución y comercialización in situ con reglas de juego uniformes.

\footnotetext{
${ }^{31}$ Empresa prestadora de energía mixta, con participación mayoritaria de la Nación, que por mandato de Ley 143 de 1994, se separó de la transportadora ISA y en la actualidad se dedica a generar y comercializar energía eléctrica.

${ }^{32}$ Volcanes.

${ }^{33}$ Fase en la que se estudian las condiciones de un reservorio geotérmico para su posible explotación.
}

REVISTA VIA INVENIENDI ET IUDICANDI, VOL. 8, No. 2 
En la actualidad la generación local no está reglamentada y su uso no está permitido debido a que la ley prohíbe a las empresas que hagan parte del sistema Interconectado nacional ejercer más de una de las actividades relacionadas con las actividades dentro de dicho sistema, excepto la comercialización que si se puede realizar con las actividades de generación y/o la distribución de energía.

Lo permitido en Colombia para el tema de despacho descentralizado hace referencia a las zonas no interconectadas, la autogeneración y la cogeneración. Siendo el primero el proceso de producción combinado de energía eléctrica y energía térmica, que hace parte integrante de la actividad productiva de quien produce dichas energías, destinadas ambas al consumo propio o de terceros en procesos industriales 0 comerciales, y que cumple los requisitos exigidos en la ley 1215 de 2008 y la autogeneración la actividad de producción de energía eléctrica exclusivamente para atender sus propias necesidades, sin usar la red pública para fines distintos al de obtener respaldo del SIN, pero no para sistemas de generación individuales por fuera de estos casos.

Debido a las limitaciones económicas por los altos costos, además de políticas y legislativas en el desarrollo de la Generación Distribuida y sobre los conflictos con los intereses de otros actores, particularmente con los operadores de red aún no se ha reconocido un modelo normativo y de políticas públicas claras para la generación descentralizada en las zonas no interconectadas, proyectando más bien un modelo actual que privilegia esquemas centralizados debido a los altos costos en la etapa de inicial de inversión de plantas menores y para lo cual se requieren incentivos tributarios de financiación, programas de investigación y desarrollo, así como certificados comerciales de energías renovables y subsidios, concesiones o reembolsos de capital.

En el tema contractual debe existir un sistema de condiciones claras y uniformes en los contratos de compraventa de energía con prerrogativas similares a las del despacho central de energía, teniendo como punto de referencias las dificultades presentadas de 
los contratos actuales de los productores independientes, pero además es necesario que los promotores y los operadores de red puedan interactuar sobre reglas que los beneficien.

Todo ello debe ir en concordancia con políticas que promuevan el desarrollo de nuevas fuentes energéticas limpias y de infraestructura de generación y distribución adecuadas para un correcto despacho.

Tomando el caso de la geotermia en Colombia hay que tener en cuenta que esta se ubica en zonas de cadenas montañosas y volcánicas ${ }^{34}$ y, por lo tanto, el desarrollo de infraestructura debe corresponder con un modelo de generación descentralizada que llegue a esas áreas de afluencia que permita la construcción de sus plantas de operación mediante incentivos tributarios y economías de escala, que la hagan sostenible y rentable a mediano plazo.

\section{La política de las Redes Inteligentes}

Como redes inteligentes para efectos de este artículo, se entenderá a una red eléctrica inteligente, similar al Internet, basada en "nodos independientes capaces de comunicarse entre ellos" 35 que por su modelo de funcionamiento cubren la medición energética además de controlar y proteger hasta los más pequeños desperdicios cuando se está transportando.

Estas redes podrían implementarse a través de una política nacional de desarrollo con el apoyo del Ministerio de Tecnologías de la Información y las Comunicaciones - TIC

\footnotetext{
${ }^{34}$ Por encima de los 3.000 metros de altura

${ }^{35}$ Comisión de Regulación de Energía y Gas, La generación distribuida y su posible integración al sistema interconectado nacional, http://apolo.creg.gov.co/Publicac.nsf/0/7128194c8ff15d7d0525785a007a71f5/\$FILE/CIRCULAR0532009\%20ANEXO.pdf, Consultado el 5 de Febrero de 2013.
} 
administrada por los entes territoriales específicos, con base en la ley 152 de 1994, articulo 4, en lo atinente a la coordinación en la planeación de los entes del orden nacional y territorial en la formulación, ejecución y evaluación de sus planes de desarrollo internos con respecto a otros entes territoriales. El cual implicaría un avance en el tema de los suministros de energía y el régimen de transporte y distribución, al menos para las zonas no interconectadas.

Aunque la mencionada ley estatutaria preceptúa la prioridad del gasto público con referencia al número de habitantes por región, valdría la pena destacar la totalidad del territorio que se encuentran en condiciones de electrificación deplorable que se ven forzados a migrar a cabeceras municipales.

Mediante una política de ejecución fuerte que incentive el tema de la unión entre los avances tecnológicos y la eficiencia del mercado energético, en principio para las zonas aledañas, podría visualizarse un futuro para la integración total del sistema interconectado nacional a través de redes inteligentes que permitieran monitorear y calcular un gasto y una necesidad exacta en el suministro de energía eléctrica, empezando por las zonas aledañas.

Mediante el control e intervención en el transporte de cargas eléctricas, la idea es administrar procesos de despacho con energías limpias a pequeña escala y contrario al modelo de despacho central, buscar una técnica que auto detecte y se acomode a la demanda en tiempo real, comunicando mecánicamente cualquier error de suministro y desconecte luces y aparatos superfluos mediante un sistema de redes inalámbricas.

De esta manera una red inteligente permitiría trabajar con modelos descentralizados que trabajan de proveedores a consumidores mediante una red computarizada donde cada punto de intersección ${ }^{36}$ de acuerdo con la predeterminación del sistema, conecta

${ }^{36}$ o Nodos.

REVISTA VIA INVENIENDI ET IUDICANDI, VOL. 8, No. 2 
al emisor con el receptor y al productor con el consumidor. Es estos sistemas de redes inteligentes la idea es que los usuarios que interactúen con la red, recibiendo y entregando energía limpia y renovable.

Esta tecnología de desintegración presume para los esquemas eléctricos un avance con base a la aparición de Internet y sobre todo de los blogs ${ }^{37}$ que interconectan enlaces, comentarios, y referencias en un esquema de información útil y que trabajarían de la misma manera para el despacho en la red y el abastecimiento de la energía.

Estos sistemas permitieran de manera muy útil medir las cantidades de descargas y entregas de energía geotérmica que este recorriendo la red de una zona no interconectada y aunque en principio resultarían costosos, nos permitirán convertirnos en un agente productor de energía primario a nivel mundial, capaz de abastecerse y reservar para Colombia y vender energía a otros países.

\section{Conclusiones.}

A nivel mundial la preocupación de las secuelas irreversibles causadas al medio ambiente y la escasez del petróleo llevaron a los estados a adquirir compromisos para encontrar nuevas fuentes de producción energética.

Al respecto Colombia tiene también compromisos vinculantes en procura de mitigar los gases efecto invernadero y encontrar sistemas de uso racional energético a través de energías alternativas renovables y sistemas de ahorro en la etapa de transporte y suministro. Por ello se han creado algunas leyes que han tratado de impulsar nuevas fuentes energéticas pero que aún se quedan cortas para incentivar la participación de las mismas dentro de la canasta energética nacional.

${ }^{37}$ OP CIT, Comisión de Regulación de Energía y Gas, La Generación Distribuida y su posible integración al Sistema Interconectado Nacional.

REVISTA VIA INVENIENDI ET IUDICANDI, VOL. 8, No. 2 
La energía geotérmica es una excelente opción, ya analizada con anterioridad y que nos abre hoy en día un mercado energético limpio con Ecuador pero que al igual que las otras fuentes de energía alternativa renovable, necesita del gobierno para su eficacia a través de una visión político energética descentralizada, que tome en cuenta las zonas que no están conectadas al sistema central de despacho de energía y que por ende necesitan con urgencia recibir un tratamiento diferente.

Ello no es posible solamente con marcos normativos, sino con políticas fuertes que incentiven el uso de nuevos métodos de provisión de energía mediante sistemas de generación descentralizados, los cuales son ideales para el aprovechamiento de fuentes limpias. Así mismo el incentivo de inversión para el monte y puesta en funcionamiento de las plantas es importante mediante incentivos tributarios de financiación, programas de investigación y mediante certificados comerciales de energías renovables, subsidios, concesiones o reembolsos de capital.

Un marco jurídico también es indispensable para lograr una igualdad de condiciones material que permita participar a todos los agentes de sistemas descentralizados y obtener ganancias, dentro de los cuales se incluyen también cuales los operadores de red.

En algunos países como Estados Unidos, mas específicamente en el estado de California, los sistemas de Generación Distribuidos son regulados y promovidos para plantas a pequeña escala con energéticos renovables, que a la vez son un respaldo de garantía a la red central en los casos de horas pico o desabastecimiento, pero que aun así no dejan de alimentar a sus respectivos usuarios locales.

Una forma de efectivizar los Sistema de Generación Distribuida es mediante avances tecnológicos que permitan monitorear suministros de despacho de pequeña escala a través de redes inteligentes para lo cual, es necesaria la colaboración del gobierno 
central a través de planes nacionales de desarrollo y la ejecución de los mismos por parte de los gobiernos locales.

Todo ello en favor de la modernización de las áreas rurales con respeto a la cultura y el habitad en que ellas se encuentran y que corresponden a un total del $65 \%$ del territorio Colombiano, en el que sus habitantes se ven en la obligación de migrar hacia las áreas urbanas.

La solución entonces a los problemas de desabastecimiento en las Zonas No Interconectadas es posible con sistemas energéticos continuos y confiables, próximos y con reglas de juego específicas y diferentes a las tradicionales llamados, Sistemas de Generación Distribuida.

\section{Referencias}

Acosta M., AMYLKAR D, Regulación Internacional de las Energías renovables y de la Eficiencia Energética, Universidad Externado de Colombia, 2011

Consorcio Energético Corpoema, Formulación de un Plan de Desarrollo para las Fuentes no Convencionales de Energía en Colombia, http://www.upme.gov.co/Sigic/DocumentosF/Vol_1_Plan_Desarrollo.pdf, consultado el 5 de febrero de 2012.

Comisión de Regulación de Energía y Gas, La generación distribuida y su posible integración al sistema interconectado nacional, http://apolo.creg.gov.co/Publicac.nsf/0/7128194c8ff15d7d0525785a007a71f5/\$FILE/CI RCULAR053-2009\%20ANEXO.pdf.

Comisión de Regulación de Energía y gas, 2003 ( 200325 de Septiembre de 2003), Resolución 073

Comisión de Regulación de Energía y Gas, 2008 Resolución 160 de 2008. 
Comisión Económica Para América Latina y el Caribe - CEPAL, 1998, Financiamiento y regulación de las fuentes de energía nuevas y renovables, El caso de la Geotermia". Naciones Unidas

http:// www.climatizaciongeotermica.com/geotermia/historia.html, consultado 20 de Febrero de 2012.

Consorcio Energético Corpoema, Formulación de un plan de Desarrollo para las Fuentes no Convencionales de Energía en Colombia, Volumen 1, página 1-1, Bogotá Diciembre de 2010

European Geothermal Energy Council, What is Geothermal? http://egec.info/about/ consultado el 12 de Abril de 2012

Instituto de Desarrollo de Recreación y Deporte - IDRD, Sinopsio

Qué y para que las políticas públicas, http://www.bogotamasactiva.gov.co/files/u1/Qu\%C3\%A9\%20y\%20Para\%20qu\%C3\%A 9\%20sirven\%20las\%20Pol\%C3\%ADticas\%20Andr\%C3\%A9\%20Noel\%20Roth.pdf, Consultado el 15 de Marzo de 2013

Ley 142 de 1994

Ley 152 de 1994

Pontificia Universidad Católica de Chile, Estudio Comparativo de las Crisis Eléctricas en Chile, California y Brasil; Aspectos Relevantes para el Nuevo Marco regulatorio Chileno, Escuela de Ingeniería http://web.ing.puc.cl/ power/paperspdf/ariztia.pdf, revisado el 26 de mayo de 2012

Proyecto Plan Nacional de Desarrollo, fuentes de energías no convencionales, volumen 2, http://www.upme.gov.co/Sigic/DocumentosF/Vol_2_Diagnostico_FNCE.pdf, Diciembre de 2012

Unidad de Planeación Minero Energética - UPME, Plan Indicativo de Expansión de Cobertura del Servicio de Energía Eléctrica http://www.upme.gov.co/Docs/Plan_Expansion_Cobertura_Energia.pdf, consultado el 15 de Agosto de 2013. 